Article

\title{
Gamma-Bazilevič Functions
}

\author{
Sa'adatul Fitri ${ }^{1}$ and Derek K. Thomas ${ }^{2, *}$
}

1 Department of Mathematics, Faculty of Mathematics and Natural Sciences, Brawijaya University, Jl. Veteran, Malang 65145, East Java, Indonesia; saadatulfitri@ub.ac.id

2 Department of Mathematics, Swansea University, Bay Campus, Swansea SA1 8EN, UK

* Correspondence: d.k.thomas@swansea.ac.uk

Received: 6 January 2020; Accepted: 29 January 2020; Published: 2 February 2020

check for updates

Abstract: For $\gamma \geq 0$ and $\alpha \geq 0$, we introduce the class $\mathcal{B}_{1}^{\gamma}(\alpha)$ of Gamma-Bazilevič functions defined for $z \in \mathbb{D}$ by $\operatorname{Re}\left\{\left[\frac{z f^{\prime}(z)}{f(z)^{1-\alpha} z^{\alpha}}+\frac{z f^{\prime \prime}(z)}{f^{\prime}(z)}+(\alpha-1)\left(\frac{z f^{\prime}(z)}{f(z)}-1\right)\right]^{\gamma}\left[\frac{z f^{\prime}(z)}{f(z)^{1-\alpha} z^{\alpha}}\right]^{1-\gamma}\right\}>0$. We shown that $\mathcal{B}_{1}^{\gamma}(\alpha)$ is a subset of $\mathcal{B}_{1}(\alpha)$, the class of $B_{1}(\alpha)$ Bazilevič functions, and is therefore univalent in $\mathbb{D}$. Various coefficient problems for functions in $\mathcal{B}_{1}^{\gamma}(\alpha)$ are also given.

Keywords: univalent; Bazilevič functions; coefficients

\section{Introduction and Definitions}

Denote by $\mathcal{A}$ the class of normalized analytic functions $f$, defined in the unit disk $\mathbb{D}$, and given by

$$
f(z)=z+\sum_{n=2}^{\infty} a_{n} z^{n}
$$

and by $\mathcal{S}$, the subclass of $\mathcal{A}$ consisting of functions which are univalent in $\mathbb{D}$.

A function $f \in \mathcal{S}$ is said to be convex if $f$ maps $\mathbb{D}$ onto a convex set, and starlike if $f$ maps $\mathbb{D}$ onto a set star-shaped with respect to the origin. Let $\mathcal{C}$ and $\mathcal{S}^{*}$ denote the classes of convex and starlike functions in $\mathcal{S}$ respectively. Then $f \in \mathcal{C}$ if and only if $\operatorname{Re}\left(1+\left(z f^{\prime \prime}(z) / f^{\prime}(z)\right)\right)>0$ for $z \in \mathbb{D}$. Similarly, $f \in \mathcal{S}^{*}$ if and only if $\operatorname{Re}\left(z f^{\prime}(z) / f(z)\right)>0$ for $z \in \mathbb{D}$.

For $\alpha \in \mathbb{R}$, the class $\mathcal{M}_{\alpha}$ of $\alpha$-convex functions defined by,

$$
\operatorname{Re}\left\{\alpha\left(1+\frac{z f^{\prime \prime}(z)}{f^{\prime}(z)}\right)+(1-\alpha)\left(\frac{z f^{\prime}(z)}{f(z)}\right)\right\}>0,
$$

for $z \in \mathbb{D}$ and $\frac{f(z)}{z} f^{\prime}(z) \neq 0$ is well known. Introduced by Miller, Mocanu and Reade [1], many interesting properties for functions in $\mathcal{M}_{\alpha}$ have been found (See e.g., [2,3]).

Denote by $\mathcal{M}^{\gamma}$ the analogue of $\mathcal{M}_{\alpha}$ in term of powers, defined for $\gamma \in \mathbb{R}$ by

$$
\operatorname{Re}\left\{\left(1+\frac{z f^{\prime \prime}(z)}{f^{\prime}(z)}\right)^{\gamma}\left(\frac{z f^{\prime}(z)}{f(z)}\right)^{1-\gamma}\right\}>0,
$$

for $z \in \mathbb{D}$. The class $\mathcal{M}^{\gamma}$ was introduced in [4], and many interesting properties of functions in $\mathcal{M}^{\gamma}$ have been found. It was shown in [4] that $\mathcal{M}^{\gamma}$ is a subset of $\mathcal{S}^{*}$. Further, sharp bounds for $\left|a_{2}\right| a_{n} d\left|a_{3}\right|$ were obtained, together with the sharp Fekete-Szegö theorem. Other result can be found in $[5,6]$.

The purpose of this paper is to introduce an analogue of $\mathcal{M}^{\gamma}$ for Bazilevič functions. We first recall the Bazilevič functions $\mathcal{B}_{1}(\alpha)$ introduced by Singh in 1973, which form a natural subset of $\mathcal{S}$ as follows [7]. 
Definition 1. Let $f \in \mathcal{A}$. Then for $\alpha \geq 0, f \in \mathcal{B}_{1}(\alpha)$ if, and only if, for $z \in \mathbb{D}$,

$$
\operatorname{Re}\left[f^{\prime}(z)\left(\frac{f(z)}{z}\right)^{\alpha-1}\right]>0 \text {. }
$$

We next introduce the Gamma-Bazilevič functions as follows, noting that we restrict our definition $\gamma \geq 0$ merely for convenience.

Definition 2. Let $f \in \mathcal{A}$, with $f(z) \neq 0$ and $f^{\prime}(z) \neq 0$. For $\gamma \geq 0$ and $\alpha \geq 0$, a function $f \in \mathcal{A}$ is said to be Gamma-Bazilevič if, for $z \in \mathbb{D}$,

$$
\operatorname{Re}\left\{\left[\frac{z f^{\prime}(z)}{f(z)^{1-\alpha} z^{\alpha}}+\frac{z f^{\prime \prime}(z)}{f^{\prime}(z)}+(\alpha-1)\left(\frac{z f^{\prime}(z)}{f(z)}-1\right)\right]^{\gamma}\left[\frac{z f^{\prime}(z)}{f(z)^{1-\alpha} z^{\alpha}}\right]^{1-\gamma}\right\}>0 .
$$

We denote this class by $\mathcal{B}_{1}^{\gamma}(\alpha)$.

Clearly $\mathcal{B}_{1}^{0}(\alpha)=\mathcal{B}_{1}(\alpha)$, and $\mathcal{B}_{1}^{\gamma}(0)=\mathcal{M}^{\gamma}$. We also note that when $\alpha=1$ and $\gamma=0$, we obtain the class $\mathcal{R}$ of functions whose derivative has a positive real part, and that when $\alpha=0$ and $\gamma=0$ we obtain the starlike functions, and when $\alpha=0$ and $\gamma=1$ we obtain the convex functions.

We also note that when $\gamma=1$, we obtain the following new class $\mathcal{B}_{1}^{\gamma}(1)$, which forms a subset of $\mathcal{R}$.

$$
\operatorname{Re}\left\{f^{\prime}(z)+\frac{z f^{\prime \prime}(z)}{f^{\prime}(z)}\right\}>0
$$

\section{Preliminaries}

We begin by stating two Lemmas which we will use in what follows.

Lemma 1 (Nunokawa, [8]). Let $p$ be analytic in $\mathbb{D}$, with $p(z) \neq 0$ and $p(0)=1$. If there exists $z_{0} \in \mathbb{D}$, such that $\left|\arg p\left(z_{0}\right)\right|<\frac{\alpha \pi}{2}$ for $|z|<\left|z_{0}\right|$, and $\left|\arg p\left(z_{0}\right)\right|=\frac{\alpha \pi}{2}$ for some $\alpha>0$, then

$$
\frac{z_{0} p^{\prime}\left(z_{0}\right)}{p\left(z_{0}\right)}=i k \alpha,
$$

where

$$
k \geq \frac{1}{2}\left(a+\frac{1}{a}\right) \quad \text { when } \quad \arg p\left(z_{0}\right)=\alpha \frac{\pi}{2},
$$

and

$$
k \leq-\frac{1}{2}\left(a+\frac{1}{a}\right) \quad \text { when } \quad \arg p\left(z_{0}\right)=-\alpha \frac{\pi}{2},
$$

and where $p^{1 / \alpha}\left(z_{0}\right)= \pm$ ia for $a>0$.

Let $\mathcal{P}$ be the class of function $h$ satisfying $\operatorname{Re} h(z)>0$ for $z \in \mathbb{D}$, with expansion

$$
h(z)=1+\sum_{n=1}^{\infty} c_{n} z^{n} .
$$

We shall use the following results concerning the coefficients $c_{n}$ of $h \in \mathcal{P}$, which can be found in [9].

Lemma 2. If $h \in \mathcal{P}$ and be given by (2), then $\left|c_{n}\right| \leq 2$ for $n \geq 1$, and

$$
\left|c_{2}-\frac{\mu}{2} c_{1}^{2}\right| \leq \max \{2,2|\mu-1|\}= \begin{cases}2, & 0 \leq \mu \leq 2, \\ 2|\mu-1|, & \text { elsewhere. }\end{cases}
$$




\section{Gamma-Bazilevič Functions}

We first show $\mathcal{B}_{1}^{\gamma}(\alpha) \subset \mathcal{B}_{1}(\alpha)$, so that functions in $\mathcal{B}_{1}^{\gamma}(\alpha)$ are univalent in $\mathbb{D}$.

Theorem 1. Let $f \in \mathcal{A}$. Then for $\gamma \geq 0$ and $\alpha \geq 0$,

$$
\operatorname{Re}\left\{\left[\frac{z f^{\prime}(z)}{f(z)^{1-\alpha} z^{\alpha}}+\frac{z f^{\prime \prime}(z)}{f^{\prime}(z)}+(\alpha-1)\left(\frac{z f^{\prime}(z)}{f(z)}-1\right)\right]^{\gamma}\left[\frac{z f^{\prime}(z)}{f(z)^{1-\alpha} z^{\alpha}}\right]^{1-\gamma}\right\}>0
$$

implies

$$
\operatorname{Re}\left\{\frac{z f^{\prime}(z)}{f(z)^{1-\alpha} z^{\alpha}}\right\}>0
$$

for $z \in \mathbb{D}$. Thus $\mathcal{B}_{1}^{\gamma}(\alpha) \subset \mathcal{B}_{1}(\alpha)$.

Proof. Let $p(z)=\frac{z f^{\prime}(z)}{f(z)^{1-a} z^{a}}$, then

$$
p(z)+\frac{z p^{\prime}(z)}{p(z)}=\frac{z f^{\prime}(z)}{f(z)^{1-\alpha} z^{\alpha}}+\frac{z f^{\prime \prime}(z)}{f^{\prime}(z)}+(\alpha-1)\left(\frac{z f^{\prime}(z)}{f(z)}-1\right) .
$$

Now note that $p(z)$ is analytic in $\mathbb{D}$ with $p(z) \neq 0$ and $p(0)=1$. Suppose that there exists a point $z_{0} \in \mathbb{D}$, such that $\left|\arg p\left(z_{0}\right)\right|<\frac{\pi}{2}$ for $|z|<\left|z_{0}\right|$ and $\left|\arg p\left(z_{0}\right)\right|=\frac{\pi}{2}$. Then by Lemma 1 ,

$$
\frac{z_{0} p^{\prime}\left(z_{0}\right)}{p\left(z_{0}\right)}=i k
$$

where

$$
k \geq \frac{1}{2}\left(a+\frac{1}{a}\right) \quad \text { when } \arg p\left(z_{0}\right)=\frac{\pi}{2},
$$

and

$$
k \leq-\frac{1}{2}\left(a+\frac{1}{a}\right) \quad \text { when } \arg p\left(z_{0}\right)=-\frac{\pi}{2},
$$

and where $p\left(z_{0}\right)= \pm i a$ for $a>0$.

There are two cases.

Case 1. If $\arg p\left(z_{0}\right)=\frac{\pi}{2}$, then

$$
\begin{aligned}
& \arg \left\{\left(p\left(z_{0}\right)+\frac{z_{0} p^{\prime}\left(z_{0}\right)}{p\left(z_{0}\right)}\right)^{\gamma} p\left(z_{0}\right)^{1-\gamma}\right\} \\
& =\gamma \arg \left[p\left(z_{0}\right)+\frac{z_{0} p^{\prime}\left(z_{0}\right)}{p\left(z_{0}\right)}\right]+(1-\gamma) \arg p\left(z_{0}\right) \\
& =\gamma \arg (i a+i k)+(1-\gamma) \frac{\pi}{2} \\
& =\gamma \frac{\pi}{2}+(1-\gamma) \frac{\pi}{2} \\
& =\frac{\pi}{2},
\end{aligned}
$$

where $p\left(z_{0}\right)=i a$ and $k \geq \frac{1}{2}\left(a+\frac{1}{a}\right)$.

Case 2. If $\arg p\left(z_{0}\right)=-\frac{\pi}{2}$, then 


$$
\begin{aligned}
& \arg \left\{\left(p\left(z_{0}\right)+\frac{z_{0} p^{\prime}\left(z_{0}\right)}{p\left(z_{0}\right)}\right)^{\gamma} p\left(z_{0}\right)^{1-\gamma}\right\} \\
& =\gamma \arg \left[p\left(z_{0}\right)+\frac{z_{0} p^{\prime}\left(z_{0}\right)}{p\left(z_{0}\right)}\right]+(1-\gamma) \arg p\left(z_{0}\right) \\
& =\gamma \arg (-i a+i k)-(1-\gamma) \frac{\pi}{2} \\
& =-\gamma \frac{\pi}{2}-(1-\gamma) \frac{\pi}{2} \\
& =-\frac{\pi}{2}
\end{aligned}
$$

where $p\left(z_{0}\right)=-i a$ and $k \leq-\frac{1}{2}\left(a+\frac{1}{a}\right)$. Therefore, we have a contradiction. There is thus no point $z_{0} \in \mathbb{D}$ such that $\left|\arg p\left(z_{0}\right)\right|<\frac{\pi}{2}$ for $|z|<\left|z_{0}\right|$, and $\left|\arg p\left(z_{0}\right)\right|=\frac{\pi}{2}$.

\section{Initial Coefficients}

We first find expressions for $a_{2}$ and $a_{3}$ in terms of the coefficients of $h \in \mathcal{P}$.

It follows from Definition 2 that we can write,

$$
\left[\frac{z f^{\prime}(z)}{f(z)^{1-\alpha} z^{\alpha}}+\frac{z f^{\prime \prime}(z)}{f^{\prime}(z)}+(\alpha-1)\left(\frac{z f^{\prime}(z)}{f(z)}-1\right)\right]^{\gamma}\left[\frac{z f^{\prime}(z)}{f(z)^{1-\alpha} z^{\alpha}}\right]^{1-\gamma}=h(z),
$$

where $h \in \mathcal{P}$.

Equating coefficients in (3) gives

$$
\begin{aligned}
& a_{2}=\frac{c_{1}}{(1+\alpha)(1+\gamma)}, \\
& a_{3}=\frac{1}{(2+\alpha)(1+2 \gamma)}\left(c_{2}-\frac{\left(\alpha^{2} \gamma^{2}-\alpha^{2} \gamma+\alpha^{2}+2 \alpha \gamma^{2}-4 \alpha \gamma+\alpha+\gamma^{2}-7 \gamma-2\right)}{2\left(1+\alpha^{2}\right)(1+\gamma)^{2}} c_{1}^{2}\right) .
\end{aligned}
$$

We now extend coefficient results given in [6] for the coefficients of $\mathcal{M}^{\gamma}$ and the results of Singh [7] for $\mathcal{B}_{1}(\alpha)$, noting that the bounds for $\left|a_{2}\right|$ and $\left|a_{3}\right|$ hold for all $\gamma \geq 0$ and $\alpha \geq 0$.

Theorem 2. If $f \in \mathcal{B}_{1}^{\gamma}(\alpha)$ and is given by (1), then

$$
\begin{aligned}
& \left|a_{2}\right| \leq \frac{2}{(1+\alpha)(1+\gamma)} \\
& \left|a_{3}\right| \leq \frac{2}{(2+\alpha)(1+2 \gamma)}
\end{aligned}
$$

when $0 \leq \gamma \leq \frac{1}{2}(7+\sqrt{57})$ and $\alpha \geq \frac{-1+4 \gamma-2 \gamma^{2}}{2\left(1-\gamma+\gamma^{2}\right)}+\frac{1}{2} \sqrt{\frac{9+12 \gamma-4 \gamma^{2}+16 \gamma^{3}}{\left(1-\gamma+\gamma^{2}\right)^{2}}}$ and when $\gamma>\frac{1}{2}(7+\sqrt{57})$.

Also

$$
\left|a_{3}\right| \leq \frac{2\left(3+\alpha+9 \gamma+8 \alpha \gamma+3 \alpha^{2} \gamma\right)}{(1+\alpha)^{2}(2+\alpha)(1+\gamma)^{2}(1+2 \gamma)}
$$

when $0 \leq \gamma<\frac{1}{2}(7+\sqrt{57})$ and $0 \leq \alpha<\frac{-1+4 \gamma-2 \gamma^{2}}{2\left(1-\gamma+\gamma^{2}\right)}+\frac{1}{2} \sqrt{\frac{9+12 \gamma-4 \gamma^{2}+16 \gamma^{3}}{\left(1-\gamma+\gamma^{2}\right)^{2}}}$ all the inequalities are sharp. 
Proof. The first inequality in Theorem 2 follows at once from (4) since $\left|c_{1}\right| \leq 2$.

For $\left|a_{3}\right|$, from (4) we use Lemma 2, and write

$$
\left|a_{3}\right|=\frac{1}{(2+\alpha)(1+2 \gamma)}\left|c_{2}-\frac{\left(\alpha^{2} \gamma^{2}-\alpha^{2} \gamma+\alpha^{2}+2 \alpha \gamma^{2}-4 \alpha \gamma+\alpha+\gamma^{2}-7 \gamma-2\right)}{2\left(1+\alpha^{2}\right)(1+\gamma)^{2}} c_{1}^{2}\right| .
$$

Then in Lemma 2, let

$$
\mu=\frac{\left(\alpha^{2} \gamma^{2}-\alpha^{2} \gamma+\alpha^{2}+2 \alpha \gamma^{2}-4 \alpha \gamma+\alpha+\gamma^{2}-7 \gamma-2\right)}{\left(1+\alpha^{2}\right)(1+\gamma)^{2}},
$$

so that applying Lemma 2 gives the inequalities for $\left|a_{3}\right|$.

The inequality for $\left|a_{2}\right|$ is sharp when $c_{1}=2$. The first inequality for $\left|a_{3}\right|$ is sharp when $c_{1}=0$ and $c_{2}=2$, and the second inequality for $\left|a_{3}\right|$ is sharp when $c_{1}=c_{2}=2$, which completes the proof of Theorem 2.

\section{Fekete-Szegö Theorem}

We next establish sharp Fekete-Szegö inequalities for $\mathcal{B}_{1}^{\gamma}(\alpha)$, which extends those given in [7] for $\mathcal{B}_{1}(\alpha)$, and in [4] for $\mathcal{M}^{\gamma}$.

Theorem 3. Let $f \in \mathcal{B}_{1}^{\gamma}(\alpha)$. Then for $v \in \mathbb{R}$,

$$
\left|a_{3}-v a_{2}^{2}\right| \leq\left\{\begin{array}{c}
\frac{2\left(3 \alpha^{2} \gamma+\alpha(-4 \gamma(v-2)-2 v+1)+\gamma(9-8 v)-4 v+3\right)}{(1+\alpha)^{2}(2+\alpha)(1+\gamma)^{2}(1+2 \gamma)} \\
\text { if } v \leq \frac{\alpha^{2}\left(-\gamma^{2}\right)+\alpha^{2} \gamma-\alpha^{2}-2 \alpha \gamma^{2}+4 \alpha \gamma-\alpha-\gamma^{2}+7 \gamma+2}{4 \alpha \gamma+2 \alpha+8 \gamma+4} \\
\frac{2}{(2+\alpha)(1+2 \gamma)}, \\
\quad \text { if } \frac{\alpha^{2}\left(-\gamma^{2}\right)+\alpha^{2} \gamma-\alpha^{2}-2 \alpha \gamma^{2}+4 \alpha \gamma-\alpha-\gamma^{2}+7 \gamma+2}{4 \alpha \gamma+2 \alpha+8 \gamma+4} \leq v \\
\leq \frac{\alpha^{2} \gamma^{2}+5 \alpha^{2} \gamma+\alpha^{2}+2 \alpha \gamma^{2}+12 \alpha \gamma+3 \alpha+\gamma^{2}+11 \gamma+4}{4 \alpha \gamma+2 \alpha+8 \gamma+4} \\
-\frac{2\left(3 \alpha^{2} \gamma+\alpha(-4 \gamma(v-2)-2 v+1)+\gamma(9-8 v)-4 v+3\right)}{(1+\alpha)^{2}(2+\alpha)(1+\gamma)^{2}(1+2 \gamma)} \\
\text { if } v \geq \frac{\alpha^{2} \gamma^{2}+5 \alpha^{2} \gamma+\alpha^{2}+2 \alpha \gamma^{2}+12 \alpha \gamma+3 \alpha+\gamma^{2}+11 \gamma+4}{4 \alpha \gamma+2 \alpha+8 \gamma+4}
\end{array} .\right.
$$

All the inequalities are sharp.

Proof. From (4) we obtain

$$
\left|a_{3}-v a_{2}^{2}\right|=\frac{2}{(2+\alpha)(1+2 \gamma)}\left|c_{2}-\frac{\mu}{2} c_{1}^{2}\right|,
$$

with

$$
\mu=\frac{-2+\alpha+\alpha^{2}-7 \gamma-4 \alpha \gamma-\alpha^{2} \gamma+\gamma^{2}+2 \alpha \gamma^{2}+\alpha^{2} \gamma^{2}+4 v+2 \alpha v+8 \gamma v+4 \alpha \gamma v}{(1+\alpha)^{2}(1+\gamma)^{2}}
$$


Applying Lemma 2, $\mu \in[0,2]$ whenever

$$
\begin{aligned}
& \frac{\alpha^{2}\left(-\gamma^{2}\right)+\alpha^{2} \gamma-\alpha^{2}-2 \alpha \gamma^{2}+4 \alpha \gamma-\alpha-\gamma^{2}+7 \gamma+2}{4 \alpha \gamma+2 \alpha+8 \gamma+4} \leq v \\
& \leq \frac{\alpha^{2} \gamma^{2}+5 \alpha^{2} \gamma+\alpha^{2}+2 \alpha \gamma^{2}+12 \alpha \gamma+3 \alpha+\gamma^{2}+11 \gamma+4}{4 \alpha \gamma+2 \alpha+8 \gamma+4},
\end{aligned}
$$

gives the second inequality.

When $\mu$ outside $[0,2]$, Lemma 2 gives the first inequality when

$$
v \leq \frac{\alpha^{2}\left(-\gamma^{2}\right)+\alpha^{2} \gamma-\alpha^{2}-2 \alpha \gamma^{2}+4 \alpha \gamma-\alpha-\gamma^{2}+7 \gamma+2}{4 \alpha \gamma+2 \alpha+8 \gamma+4},
$$

and the third inequality when

$$
v \geq \frac{\alpha^{2} \gamma^{2}+5 \alpha^{2} \gamma+\alpha^{2}+2 \alpha \gamma^{2}+12 \alpha \gamma+3 \alpha+\gamma^{2}+11 \gamma+4}{4 \alpha \gamma+2 \alpha+8 \gamma+4}
$$

The second inequality is sharp when $c_{1}=0$ and $c_{2}=2$. The first and third inequalities are sharp when $c_{1}=c_{2}=2$. This completes the proof of Theorem 3 .

\section{Logarithmic Coefficients}

The logarithmic coefficients $g_{n}$ of $f$ are defined in $\mathbb{D}$ by

$$
\log \frac{f(z)}{z}=2 \sum_{n=1}^{\infty} g_{n} z^{n}
$$

Differentiating (5) and equating coefficients gives

$$
\begin{gathered}
g_{1}=\frac{1}{2} a_{2} \\
g_{2}=\frac{1}{2}\left(a_{3}-\frac{1}{2} a_{2}^{2}\right), \\
g_{3}=\frac{1}{2}\left(a_{4}-a_{2} a_{3}+\frac{1}{3} a_{2}^{3}\right) .
\end{gathered}
$$

For $f \in \mathcal{B}_{1}^{\gamma}(\alpha)$, we give sharp bounds for $\left|g_{n}\right|$ when $n=1,2$, which extend those given in [10] and [6].

Theorem 4. Let $f \in \mathcal{B}_{1}^{\gamma}(\alpha)$, then

$$
\begin{gathered}
\left|g_{1}\right| \leq \frac{1}{(1+\alpha)(1+\gamma)} \text { when } \gamma \geq 0 \text { and } \alpha \geq 0, \\
\left|g_{2}\right| \leq \frac{1}{(2+\alpha)(1+2 \gamma)}, \text { when } 0 \leq \gamma \leq 3 \text { and } \alpha \geq-1+\sqrt{\frac{1+2 \gamma}{1-\gamma+\gamma^{2}}},
\end{gathered}
$$

and when $\gamma>3$ and $\alpha \geq 0$.

Further,

$$
\left|g_{2}\right| \leq \frac{1+\left(5+6 \alpha+3 \alpha^{2}\right) \gamma}{(1+\alpha)^{2}(2+\alpha)(1+\gamma)^{2}(1+2 \gamma)}, \text { when } 0<\gamma<3 \text { and } 0 \leq \alpha<-1+\sqrt{\frac{1+2 \gamma}{1-\gamma+\gamma^{2}}}
$$


All the inequalities are sharp.

Proof. We note first that since $\left|c_{1}\right| \leq 2$, the inequality $\left|g_{1}\right| \leq \frac{1}{(1+\alpha)(1+\gamma)}$ is trivial.

The result for $\left|g_{2}\right|$ follows at once from the above Fekete-Szegö theorem in the case $\mu=1 / 2$. For the first inequality, we use the second inequality in Theorem 3 , and for the second inequality we use the first inequality in Theorem 3.

We note that the inequality for $\left|g_{1}\right|$ is sharp when $c_{1}=2$. The first inequality for $\left|g_{2}\right|$ is sharp when $c_{2}=2$ and $c_{1}=0$, and the second inequality is sharp when choosing $c_{1}=c_{2}=2$. This completes the proof of Theorem 4 .

Remark 1. Finding sharp upper bounds for $\left|g_{n}\right|$ for all $n \geq 3$ when $f \in \mathcal{B}_{1}^{\gamma}(\alpha)$ remains an open problem. In the case $\alpha=0$, sharp results for $n=1,2,3$ have been obtained in [6]. For $\gamma=0$, it was shown in [10] that

$$
\left|g_{n}\right| \leq \frac{1}{n+\alpha}
$$

for $n=1,2,3$.

\section{Inverse Coefficients}

For any univalent function $f$ there exists an inverse function $f^{-1}$ defined on some disc $|\omega|<r_{0}(f)$, with Taylor expansion

$$
f^{-1}(\omega)=\omega+A_{2} \omega^{2}+A_{3} \omega^{3}+A_{4} \omega^{4}+\ldots
$$

Suppose that $\mathcal{B}_{1}^{\gamma}(\alpha)^{-1}$ is the set of inverse functions $f^{-1}$ of $\mathcal{B}_{1}^{\gamma}(\alpha)$, given by (6). Then $f\left(f^{-1}(\omega)\right)=$ $\omega$, and equating coefficients gives

$$
\begin{aligned}
& A_{2}=-a_{2}, \\
& A_{3}=2 a_{2}^{2}-a_{3} .
\end{aligned}
$$

We prove the following, noting again that the inequalities for $\left|A_{2}\right|$ and $\left|A_{3}\right|$ hold for all $\gamma \geq 0$ and $\alpha \geq 0$ thus extending results extend in [10] and [6].

Theorem 5. Let $f \in \mathcal{B}_{1}^{\gamma}(\alpha)$ and $f^{-1}$ be given by (6), then

$$
\begin{aligned}
& \left|A_{2}\right| \leq \frac{2}{(1+\alpha)(1+\gamma)} \\
& \left|A_{3}\right| \leq \frac{2}{(2+\alpha)(1+2 \gamma)}
\end{aligned}
$$

when $0 \leq \gamma \leq \frac{1}{2}(5+\sqrt{41})$ and $\alpha \geq \frac{1-4 \gamma-2 \gamma^{2}}{2\left(1+5 \gamma+\gamma^{2}\right)}+\frac{1}{2} \sqrt{\frac{17+92 \gamma+124 \gamma^{2}+16 \gamma^{3}}{\left(1+5 \gamma+\gamma^{2}\right)^{2}}}$,

and when $\gamma>\frac{1}{2}(5+\sqrt{41})$.

Further,

$$
\left|A_{3}\right| \leq \frac{10+6 \alpha+14 \gamma-6 \alpha^{2} \gamma}{(1+\alpha)^{2}(2+\alpha)(1+\gamma)^{2}(1+2 \gamma)}
$$

when $0 \leq \gamma<\frac{1}{2}(5+\sqrt{41})$ and $0 \leq \alpha<\frac{1-4 \gamma-2 \gamma^{2}}{2\left(1+5 \gamma+\gamma^{2}\right)}+\frac{1}{2} \sqrt{\frac{17+92 \gamma+124 \gamma^{2}+16 \gamma^{3}}{\left(1+5 \gamma+\gamma^{2}\right)^{2}}}$.

All the inequalities are sharp. 
Proof. We again use the expressions for the coefficients given in (4).

Since $(1+\alpha)(1+\gamma) a_{2}=c_{1}$ and $\left|c_{1}\right| \leq 2$, the first inequality is trivial.

Next we note that from (4)

$$
\left|A_{3}\right|=\frac{1}{(2+\alpha)(1+2 \gamma)}\left|c_{2}-\frac{\left(\alpha^{2} \gamma^{2}-\alpha^{2} \gamma+\alpha^{2}+2 \alpha \gamma^{2}+4 \alpha \gamma+5 \alpha+\gamma^{2}+9 \gamma+6\right)}{2(1+\alpha)^{2}(1+\gamma)^{2}} c_{1}^{2}\right| .
$$

Let

$$
\mu=\frac{\left(\alpha^{2} \gamma^{2}-\alpha^{2} \gamma+\alpha^{2}+2 \alpha \gamma^{2}+4 \alpha \gamma+5 \alpha+\gamma^{2}+9 \gamma+6\right)}{(1+\alpha)^{2}(1+\gamma)^{2}}
$$

and applying Lemma 2 gives the required inequalities.

The inequality for $\left|A_{2}\right|$ is sharp when $c_{1}=2$. The first inequality for $\left|A_{3}\right|$ is sharp on choosing $c_{1}=0$ and $c_{2}=2$, and the second inequality is sharp when $c_{1}=c_{2}=2$. This completes the proof of Theorem 5 .

Remark 2. Clearly finding sharp bounds for $\left|a_{4}\right|$ and $\left|A_{4}\right|$ appears to be far more difficult, and requires significantly more analysis. We note that applying the often used lemmas in [9] fails to give sharp results.

We also note that even when $\gamma=1$, the analysis for $\left|a_{4}\right|$ and $\left|A_{4}\right|$ is far from simple, and appears to require methods deeper than those used or mentioned in this paper.

Author Contributions: All authors have read and agreed to the published version of the manuscript.

Funding: This research received no external funding.

Conflicts of Interest: The authors declare no conflict of interest.

\section{References}

1. Miller, S.S.; Mocanu, P.T.; Reade, M.O. All $\alpha$-convex functions are starlike. Rev. Roumaine Math. Pures Appl. 1972, 17, 1395-1397.

2. Kulshrestha, P.K. Coeffcients for alpha-convex univalent functions. Bull. Am. Math. Soc. 1974, 80, 341-342. [CrossRef]

3. Miller, S.S.; Mocanu, P.T.; Reade, M.O. All $\alpha$-convex functions are univalent and starlike. Proc. Am. Math. Soc. 1973, 37, 553-554. [CrossRef]

4. Darus, M.; Thomas, D.K. $\alpha$-Logarithmically Convex Functions. Indian J. Pure Appl. Math. 1998, 29, $1049-1059$.

5. Darus, M.; Thomas, D.K. On the Coefficients of $\alpha$-Logarithmically Convex Functions. Jũ̄anäbha 2015, 15, 31-36.

6. Thomas, D.K. On the Coefficients of Gamma-Starlike Functions. J. Korean Math. Soc. 2018, 55, $175-184$.

7. Singh, R. On Bazilevič Functions. Proc. Am. Math. Soc. 1973, 38, 261-271.

8. Nunokawa, M. On the order of strongly starlikeness of strongly convex functions. Proc. Jpn. Acad. Ser. A Math. Sci. 1993, 69, 234-237. [CrossRef]

9. Ali, R.M. Coefficients of the Inverse of Strongly Starlike Functions. Bull. Malays. Math. Sci. Soc. 2003, 26, 63-71.

10. Thomas, D.K. On the Coefficients of Bazilevič Functions with Logarithmic Growth. Indian J. Math. 2015, 57, 403-418.

(C) 2020 by the authors. Licensee MDPI, Basel, Switzerland. This article is an open access article distributed under the terms and conditions of the Creative Commons Attribution (CC BY) license (http:/ / creativecommons.org/licenses/by/4.0/). 